\title{
A NEW UPPER BOUND FOR THE MINIMUM OF AN INTEGRAL LATTICE OF DETERMINANT 1
}

\author{
J. H. CONWAY AND N. J. A. SLOANE
}

\begin{abstract}
Let $\Lambda$ be an $n$-dimensional integral lattice of determinant 1 . We show that, for all sufficiently large $n$, the minimal nonzero squared length in $\Lambda$ does not exceed $[(n+$ $6) / 10]$. This bound is a consequence of some new conditions on the theta series of these lattices; these conditions also enable us to find the greatest possible minimal squared length in all dimensions $n \leq 33$. In particular, we settle the "no-roots" problem: There is a determinant 1 lattice containing no vectors of squared length 1 or 2 precisely when $n \geq 23, n \neq 25$. There are also analogues of all these results for codes.
\end{abstract}

\section{INTRODUCTION}

The problem of classifying $n$-dimensional integral lattices of determinant 1 has been studied by Magnus, Mordell, Ko, Witt, Kneser, Niemeier and others [4, Chapters 1, 16, and 17]. The lattices $\Lambda$ of this type for which the minimal norm

$$
\min \{u \cdot u: u \in \Lambda, u \neq 0\}
$$

takes its highest possible value $\mu$ are of the greatest interest. It was shown in [7] that for even lattices (those in which $u \cdot u$ is always even), the minimal norm is at most $2[n / 24]+2$, while for odd lattices (those in which $u \cdot u$ is sometimes odd) the corresponding bound is $[n / 8]+1[7,11]$. These are the bounds one would expect from the dimension of the space of available theta series. In fact, it is known that $\mu$ differs from these bounds by an amount that tends to infinity with $n$, so that equality can hold for only finitely many lattices [7]. In the odd case, the bound holds with equality for precisely 12 lattices, the highest dimension of which is 23 [2, 4, Chapter 19]. As to lower bounds, it is known that both even and odd lattices exist in which the minimal norm is asymptotically at least $n / 2 \pi e \quad[4$, Chapter $7 ; 10]$.

Received by the editors October 3,1989 and, in revised form, April 10, 1990.

1980 Mathematics Subject Classification (1985 Revision). Primary 11E25, $11 \mathrm{E} 41,11 \mathrm{H} 31,52 \mathrm{~A} 45,94 \mathrm{~B} 05$. 
The purpose of this paper is to announce the following improved bound.

Theorem 1. For all sufficiently large $n$, we have $\mu \leq[(n+6) / 10]$.

(An upper bound asymptotic to $n / 9.793 \ldots$ is implied by the Kabatiansky-Levenshtein sphere-packing bound [4, Chapter 9; 5].) We believe that for odd lattices the bound of Theorem 1 in fact holds for all dimensions $n$ except 1, 2, 3, 12, 23 and 32, where special circumstances permit $\mu$ to exceed the bound by 1 .

In particular cases we can often obtain additional information. For dimensions 1 through 33, we have been able to find the exact value of $\mu$.

Theorem 2. $\mu=1$ for $n=1$ to 7,9 to 11 and $13 ; \mu=2$ for $n=8,12,14$ to 22 and $25 ; \mu=3$ for $n=23,26$ to 31 and 33; and $\mu=4$ for $n=24$ and 32 .

We also have information about the optimal lattices (those whose minimal norm equals $\mu$ ). For example, there are precisely five odd optimal lattices in 32 dimensions, while there are more than $8 \times 10^{20}$ optimal lattices (which are necessarily odd) in 33 dimensions!

Vectors of norms 1 or 2 in a lattice of determinant 1 are called roots (the reflections in such vectors are symmetries of the lattice).

Theorem 3. Determinant 1 lattices with no roots exist precisely for $n \geq 23, n \neq 25$.

Most of these theorems have analogues for binary self-dual codes.

Theorem 4. For all $n \geq 50$, the minimal distance $d$ of a self-dual code of length $n$ satisfies $d \leq 2[(n+6) / 10]$.

We can show that, for self-dual codes in which the weights are not all multiples of 4 , the bound of Theorem 4 holds for all lengths $n$ except 2, 12, 22 and 32. Here, however, the bound of McEliece et al. [6, Chapter 17;9] is asymptotically stronger, yielding $d \leq$ $0.182 n+o(n)$.

Theorem 5. The greatest minimal distance of any self-dual code of length $n$ is 2 for $n=2,4,6$ and 10; 4 for $n=8$ and 12 to 20; 6 for $n=22,26,28,30$ and $34 ; 8$ for $n=24,32$ and 36 to 44; 10 for $n=46,50,52,54$ and 58; and 12 for $n=48,56$ and 60 . 
Theorem 6. Self-dual codes with minimal distance

$d \geq 6$ exist precisely for $n \geq 22$,

$d \geq 8$ exist precisely for $n=24$ and 32 and $n \geq 36$, and

$d \geq 10$ exist precisely for $n \geq 46$.

There are precisely three self-dual codes with $n=32, d=8$ such that not all weights are multiples of 4 . (In the case where the weights are multiples of 4 , it was already known that there are precisely 5 codes [3].)

\section{REMARKS ON THE PROOFS}

Theorem 2 follows from a detailed study of the theta series and by explicit constructions in dimensions $n \leq 32$, while Theorem 3 also uses an analytic argument (involving the average theta series) for $n \geq 33$. We now sketch the proof of Theorem 1. Complete details will appear elsewhere.

Let $\Lambda$ be an $n$-dimensional integral lattice of determinant 1 . If $\Lambda$ is even, the result follows from [7], so we assume $\Lambda$ is odd. The theta series $\Theta_{\Lambda}(q)=\sum_{u \in \Lambda} q^{u^{*} u}$ can be written as

$$
\Theta_{\Lambda}(q)=\sum_{j=0}^{[n / 8]} a_{j} \quad \Delta_{8}(q)^{j} \quad \theta_{3}(q)^{n-8 j},
$$

where

$$
\Delta_{8}(q)=\prod_{m=1}^{\infty}\left(1-q^{2 m-1}\right)^{8}\left(1-q^{4 m}\right)^{8}
$$

[4, page 187]. $\left(\theta_{2}, \theta_{3}\right.$ and $\theta_{4}$ are the usual Jacobi theta series [4, page 102;12, page 464]. If $\Lambda$ has minimal norm at least $\sigma$, then $a_{0}, \ldots, a_{\sigma-1}$ are determined and can be found from the Bürmann-Lagrange theorem $[7 ; 8 ; 12$, page 128]. We obtain

$$
a_{j}=-\frac{n}{j !}\left[\frac{d^{j-1}}{d q^{j-1}}\left\{\theta_{3}^{\prime}(q) \theta_{3}(q)^{8 j-n-1} h(q)^{j}\right\}\right]_{q=0},
$$

where $h(q)=q \Delta_{8}(q)^{-1}$ (cf. [7, Equation (6); 8, page 191]).

Suppose, seeking a contradiction, that $\sigma=[(n+6) / 10]+1$. Let $n=10 k+\delta,-6 \leq \delta \leq 3$, so $\sigma=k+1$. We use (1) and the saddle point method (as in Lemma 1 of [7]) to obtain

$$
a_{k} \sim-\frac{c_{1}}{\sqrt{k}} c_{2}^{k}, \quad \text { as } k \rightarrow \infty,
$$

where $c_{2}=14.91050$ and $c_{1}$ is a positive number (depending on $\delta)$. 
We now obtain a second estimate for $a_{k}$, incompatible with (2). Let $\Lambda_{0}$ denote the even sublattice of $\Lambda$, of index 2 . The dual lattice $\Lambda_{0}^{*}$ is the union of four cosets of $\Lambda_{0}$, say $\Lambda_{0}^{*}=\bigcup_{i=0}^{3} \Lambda_{0}^{(i)}$, with $\Lambda_{0}=\Lambda_{0}^{(0)}, \Lambda=\Lambda_{0}^{(0)} \cup \Lambda_{0}^{(2)}$. We set $\Omega=\Lambda_{0}^{(1)} \cup \Lambda_{0}^{(3)}$. The theta series of $\Omega$ is given by [4, page 440, Equations (5) and (6)]:

$$
\Theta_{\Omega}(q)=\sum_{j=0}^{[n / 8]} \frac{(-1)^{j}}{16^{j}} a_{j} \theta_{4}\left(q^{2}\right)^{8 j} \theta_{2}(q)^{n-8 j}=\Sigma \beta_{r} q^{r} \quad \text { (say). }
$$

Note that the values of $r$ in (3) are rational numbers congruent to $n / 4(\bmod 2)$.

For two distinct pairs $\pm u, \pm v \in \Omega$ we cannot have $N(u)+$ $N(v)<\sigma$, since $u \pm v \in \Lambda$. This principle implies that there is at most one nonzero $\beta_{r}$ for $r<(\sigma+2) / 2$, that $\beta_{r}=0$ for $r<\sigma / 4, \beta_{r}=0$ or 2 for $r<\sigma / 2$ and (by consideration of inner products) that $\beta_{r} \leq 2 n$ for $r<(\sigma+1) / 2, n \neq 3$. (R. E. Borcherds [1] used similar ideas in studying lattices in dimensions 25 to 27.)

Thus the values of $\beta_{r}$ for $r<(\sigma+1) / 2$ are small. A second application of the Bürmann-Lagrange theorem now enables us to determine $a_{[n / 8]}, a_{[n / 8]-1}, \ldots, a_{k}$. Again applying Lemma 1 of [7], we obtain an upper bound for $a_{k}$ which is asymptotic to

$$
\frac{c_{3}}{\sqrt{k}} c_{4}^{k}, \quad \text { as } k \rightarrow \infty,
$$

where $c_{3}$ is positive and independent of $k$, and $c_{4}=7.10716 \ldots$. Comparison of (2) and (4) yields the desired contradiction.

\section{REFERENCES}

1. R. E. Borcherds, The Leech lattice and other lattices, Ph.D. dissertation, Univ. of Cambridge, 1984.

2. J. H. Conway, A. M. Odlyzko, and N. J. A. Sloane, Extremal self-dual lattices exist only in dimensions 1 to 8,12,14, 15, 23 and 24, Mathematika 25 (1978), 36-43.

3. J. H. Conway and V. Pless, On the enumeration of self-dual codes, J. Combin. Theory Ser. A 28 (1980), 26-53.

4. J. H. Conway and N. J. A. Sloane, Sphere packings, lattices and groups, Springer-Verlag, New York, 1988.

5. G. A. Kabatiansky and V. I. Levenshtein, Bounds for packings on a sphere and in space, (in Russian), Problemy Peredachi Informatsii 14 (1) (1978), 3-25.

6. F. J. MacWilliams and N. J. A. Sloane, The theory of error-correcting codes, North-Holland, Amsterdam, 1977.

7. C. L. Mallows, A. M. Odlyzko, and N. J. A. Sloane, Upper bounds for modular forms, lattices, and codes, J. Algebra 36 (1975), 68-76. 
8. C. L. Mallows and N. J. A. Sloane, An upper bound for self-dual codes, Inform. and Control 22 (1973), 188-200.

9. R. J. McEliece, E. R. Rodemich, H. C. Rumsey, Jr., and L. R. Welch, New upper bounds on the rate of a code via the Delsarte-MacWilliams inequalities, IEEE Trans. Inform. Theory 23 (1977), 157-166.

10. J. Milnor and D. Husemoller, Symmetric bilinear forms, Springer-Verlag, New York, 1973.

11. C. L. Siegel, Berechnung von Zetafunktionen an ganzzahligen Stellen, Göttingen Nach. 10 (1969), 87-102. (Gesam. Abh. vol. IV, pp. 82-97.)

12. E. T. Whittaker and G. N. Watson, A course of modern analysis, 4th ed., Cambridge Univ. Press, 1963.

Mathematics Department, Princeton University, Princeton, New JERSEY 08540

Mathematical Sciences Research Center, AT\&T Bell laboratories, Murray Hill, New Jersey 07974 
